\title{
EXPERIMENTAL EVALUATION OF AN ELECTRO-HYDROSTATIC ACTUATOR FOR SUBSEA APPLICATIONS IN A HYPERBARIC CHAMBER
}

\author{
João Pedro Duarte da Silva ${ }^{2}$, Amadeu Plácido Neto ${ }^{2}$, Victor Juliano De Negri ${ }^{1}$, Alexandre \\ Orth $^{2}$ \\ ${ }^{I}$ Federal University of Santa Catarina (UFSC), Laboratory of Hydraulic and Pneumatic Systems (LASHIP), \\ Florianópolis, Santa Catarina, Brazil. \\ ${ }^{2}$ Bosch Rexroth AG, Lohr am Main, Germany. \\ * Corresponding author: Tel.: +55 48 996841944; E-mail address: joao.pedro.d.silva@posgrad.ufsc.br
}

\begin{abstract}
A novel Electro-Hydrostatic Actuator (EHA) prototype - designed to operate on subsea gate valves in deep and ultra-deep water - is analysed and qualified in terms of functionality under design and normative constraints. The prototype is assembled in a test bench for load control in a hyperbaric chamber where the high subsea environmental pressure can be emulated. The process variables under evaluation are monitored through a set of pressure and position sensors, which are part of the prototype design. The experimental results demonstrate a robust behaviour of the actuator concerning the imposed external pressure and load forces even with a forced limitation in its power input. Moreover, the prototype performs consistently throughout the entire endurance trial, asserting high reliability. With the results obtained, the subsea EHA concept is effectually eligible to a technology readiness level 4, according to the API $17 \mathrm{~N}$.
\end{abstract}

Keywords: Electro-hydrostatic actuator, Subsea production systems, Hyperbaric test, Ultra-deep water, Verification and validation

\section{INTRODUCTION}

In the context of subsea oil \& gas production systems, there is an ongoing transition to AllElectric Systems (AES). Particularly for subsea actuators, this trend has surged due to their several advantages in comparison with the conventional hydraulic and electrohydraulic counterparts, such as installation and operational costs reduction, fast system response, reduction of umbilical diameter, high level of operational flexibility and environment-friendly design [1] [2]. Yet, many AES actuator solutions rely on electromechanical principle, which brings along substantial drawbacks. Namely, low powerdensity, cumbersome fail-safe mechanisms - if any - and higher system complexity, compromising reliability.

The application of an electro-hydrostatic actuator (EHA) in subsea production systems is a solution that can be seamlessly implemented in AESs preserving all of its good attributes aforementioned and adding the inherent advantages of conventional electro-hydraulic systems, such as reliability, compactness, robustness, high power density, high load capacity, low maintenance, simple and effective overload protection and uncomplicated commissioning [3]. All the while, it prevents the drawbacks found in the current electromechanical actuators.

In the oil and gas industry EHAs are already utilized on topsides and onshore systems, however, the adaptation of this concept for subsea application is entirely novel, which highlights the importance of its validation in hyperbaric environment. This actuator principle is already a mature technology meeting the most rigorous safety requirements in aircraft applications, where these systems are referred as "power-bywire' type of actuation systems [4].

All oil and gas equipment intended to be applied in the bathypelagic zone - located in depths between 1830 to 3000 meters - must be designed accounting the harsh environmental conditions, such as the high external pressures 
due to the water column and the difficulty to perform maintenance in such places. Beyond these considerations, in order to reduce operating costs, the equipment must operate with high availability and minimum maintenance during the entire service life of a field, which can be 25 years or more [5] [6]. To ensure this suitability, a series of international standards and norms have been issued, containing extensive qualification tests targeting all subsea-rated equipment.

In this paper, a novel EHA for subsea valves henceforth designated as subsea valve actuator (SVA) - is evaluated according to these qualification requirements, particularly in respect to the hyperbaric conditions. The precise standards applicable for subsea actuators that are considered in this evaluation are the ISO 10423, ISO 13628-4 and the API 17F. To achieve this objective, first the test subject is briefly described. Then, a test methodology is presented and, finally, the results obtained are discussed in respect to the qualification requirements present in the standards.

\section{ELECTRO-HYDROSTATIC ACTUATOR PROTOTYPE DESCRIPTION}

The subsea valve actuator prototype studied in this research is an electro-hydrostatic actuator (EHA) devised to operate with 2 inches gauge gate valves at depths of up to 3000 meters. Its simplified composition, disregarding most of the redundancies and auxiliary features, is illustrated in Figure 1 and described next.

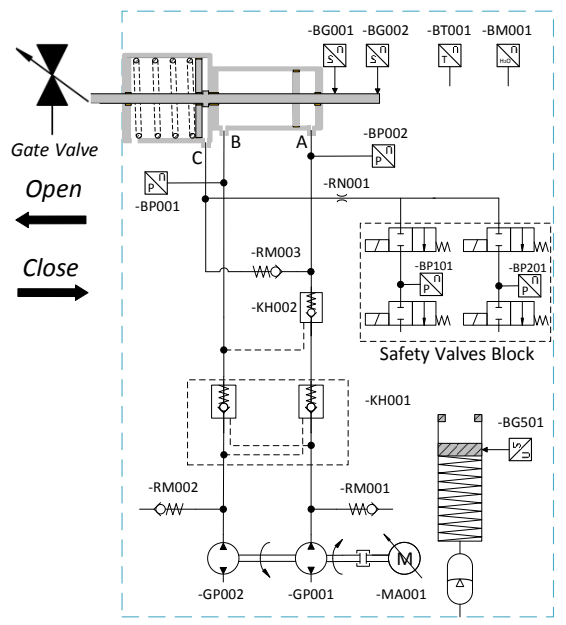

Figure 1: Schematic diagram of the SVA prototype
The components illustrated within the blue dashed rectangle in Figure 1 are all submerged in hydraulic fluid - contained inside the hull of the actuator that serves as the hydraulic reservoir as well. The electric servomotor, designated MA001 in the schematic, is coupled to two hydraulic pumps (GP001 and GP002) by a gear transmission. This drive system is responsible for the regular actuation function, moving the fourchamber hydraulic cylinder by controlling the flow rate through its chambers (A, B and C).

The four-chamber hydraulic cylinder can be divided as a spring cylinder and the work cylinder. The spring cylinder, which houses the spring, has a piston unattached to its rod, which enables it to move freely in the forward direction. For the returning direction, there is a mechanical contact between piston and rod, which does not allow the spring cylinder piston to move freely in the closing direction once it reaches this contact. The spring release occurs once the ON-OFF solenoids of the safety valves are de-energized, allowing the flow rate from chamber $\mathrm{C}$ to the reservoir. The flow restrictor (RN001) guarantees a smooth and controlled return. This mechanism constitutes the fail-safe close function of the SVA. During the regular actuation function, the spring cylinder is clamped and the ON-OFF safety valves are closed, while the gate valve movement is driven solely by the work cylinder.

The system also contains check valves (RM001 and RM002) to prevent low pressure in the hydraulic lines, pilot operated check valves (KH001 and KH002) to latch the position of the work cylinder when the drive system is at rest, another check valve (RM003) which allows the flow rate to fill the cylinder chamber $\mathrm{C}$, locking the spring compressed. Finally, the pressure inside of the SVA hull is compensated and pressurized to be constantly higher - from about 0.5 to $2 \mathrm{bar}$ - than the environmental pressure by action of several pressure compensation units in parallel. The additional pressure is generated by the preloaded springs, which compresses the piston of each compensator, as illustrated in Figure 2. The purpose is to avoid any ingress contamination from the environment such as seawater, sand, or organic materials in case of an external leakage. This constitutes the external pressure compensation function. 


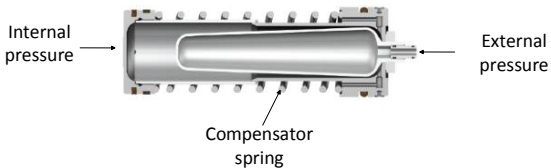

Figure 2: Pressure compensator system.

The SVA is also instrumented with a series of sensors to monitor and assess the state of its subsystems. Namely, the work cylinder is equipped with two position encoders (BG001 and BG002) and two absolute pressure sensors (BP001 and BP002). There are two additional pressure sensors between the safety valves (BP101 and BP201), one position encoder in one of the pressure compensation units (BG501) to assess approximately the available volume for compensation, and, in contact with the hydraulic medium, there are a temperature sensor and a water contamination sensor (BT001 and BM001 respectively). In addition, the motor can have its velocity, electric current consumption and winding voltages externally monitored.

The SVA control interface is solely comprised of an electrical connector, which enables an external controller unit to read its instruments, and control both the servomotor and solenoid valves.

By means of these subsystems, the scope of the functional validation of the SVA can be delineated by the following three main functions:

- Regular actuation function;

- Fail-safe close function;

- External pressure compensation function;

\section{EXPERIMENTAL METHODOLOGY}

The methodology used to evaluate the SVA is based off in the systematic approach by Kleppmann [7]. This approach consists of initially describing the test subject - as has been done in the previous section - followed by the definition of the test objectives, the test procedure description, the test bench description and finally the results interpretation.

\subsection{Test objective}

Within this approach, the test objective is to fulfil the qualification requirements regarding hyperbaric environment of the relevant standards. In addition, design requirements of the prototype relevant in hyperbaric environment that are not required by the standards are also listed for evaluation. All of the considered standards required that for the design validation of subsea actuation systems, hydraulic actuators shall be submitted to a functional test in a hyperbaric environment. ISO 10423 [8] particularly specifies the load to be either a valve/choke or a fixture that emulates the opening/closing dynamic force profile of a valve/choke with a specific well pressure differential. API 17F [9] specifies minimum rates of pressurization and depressurization of the hyperbaric chamber. The number of functional cycles is divergent in each standard, thus the harshest requirement is adopted as objective.

A summary of the test requirements is given as follows:

- Submit the equipment in a hyperbaric condition of $110 \%$ of the maximum rated depth for at least 6 hours, without compromising its functionality;

- 200 regular actuation cycles at hyperbaric condition of $110 \%$ of the maximum rated depth, with 10000 psi differential pressure in the valve fixture;

- 400 regular actuation cycles at atmospheric pressure, without load;

- 3 fail-safe cycles at hyperbaric condition of $110 \%$ of the maximum rated depth, with 10000 psi differential pressure in the valve fixture;

- 3 fail safe cycles at hyperbaric condition of $110 \%$ of the maximum rated depth, without load;

- 3 fail safe cycles at atmospheric pressure, with 10000 psi differential pressure in the valve fixture;

- 3 fail safe cycles at atmospheric pressure, without load;

- 3 pressurization-depressurization cycles from atmospheric to $110 \%$ of the maximum rated depth with minimum pressurization rate of $24 \mathrm{bar} / \mathrm{min}$ and depressurization of $36 \mathrm{bar} / \mathrm{min}$;

The required pressure differential in the valve fixture disregards the downstream pressure, which is caused by the weight from the column of fluid. In practice, the pressure differential should be considerably lower, giving the load in this test condition a substantial safety factor. Furthermore, the well pressure in a field tends to decrease as the production advances, which would render a decreasing load force through the 
cycles. This is not considered, constituting, in this sense as well, a test condition more severe than the real application.

To comply additional design constraints, all cycles must be performed with a power input limitation of $250 \mathrm{~W}$ in the SVA drive. This affects the system, as the actuation velocity is controlled to perform a full stroke in 60 seconds (disregarding the time to latch the spring cylinder). Moreover, the fail-safe cycles must finish the closing motion in less than 30 seconds.

\subsection{Test procedure}

The test shall consist of devising a device to produce a controllable force that can be mechanically coupled to the SVA stem, serving as the valve fixture. The force in such a fixture shall have to emulate the same forces involved in a real subsea actuator and gate valve interaction. The main forces involved in such assembly are delineated in Figure 3.

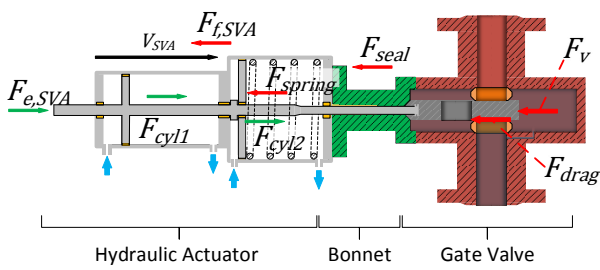

Figure 3: Force diagram in a subsea gate valve/actuator assembly.

Forces present on the actuator:

- $F_{e, S V A}$ : Enclosure force, caused by the reservoir pressure;

- $F_{c y l 1} \quad$ : Work cylinder hydraulic force;

- $F_{c y l 2}$ : Spring cylinder hydraulic force;

- $F_{\text {spring }}$ : Fail-safe spring force;

- $F_{f, S V A}$ : SVA friction forces.

Forces present on the gate valve and bonnet:

- $F_{v} \quad$ : Force acting on the stem due to the valve internal pressure;

- $F_{\text {drag }}$ : Friction force between the gate and seats of the valve;

- $F_{\text {seal }}:$ Friction force due to the contact between the seals and the stem in the bonnet.

The resultant force is then the sum of the three mentioned forces present on the gate valve and bonnet. This force profile is a function of the valve position, as the normal force in the gate valve changes dramatically once the gate valve starts to open. The normal force influences the friction force between the gate and seats of the valve [6] [10]. The load force profile applied to the SVA during the tests is illustrated in Figure 4. The steepness in which the drag force changes depends on the valve geometry and discharge factor.

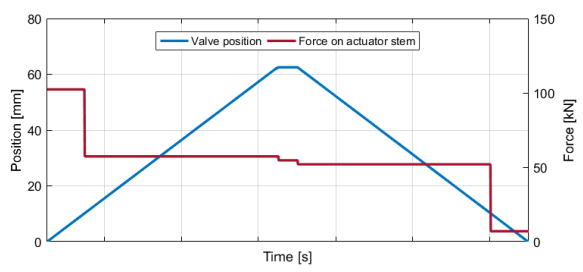

Figure 4: Force profile acting on the actuator stem as a position function, according to standard test conditions.

The load force profile of Figure 4 emulates a 2 inches gate valve with wellbore pressure of 10000 psi (690 bar) in test condition where the pressure differential through the gate decreases abruptly once the valve starts to open [6] [11]. It can be noticed that the maximum required force to move the gate valve occurs at the beginning of the movement while the valve is still closed and its pressure differential is maximum. This force is equal to $102.3 \mathrm{kN}$ considering the parameters of the gate valve taken. The minimum force occurs after closing the valve when its pressure differential is maximum again. The position where the valve starts to open is referred as crackopen position, and can be better visualized in Figure 5.

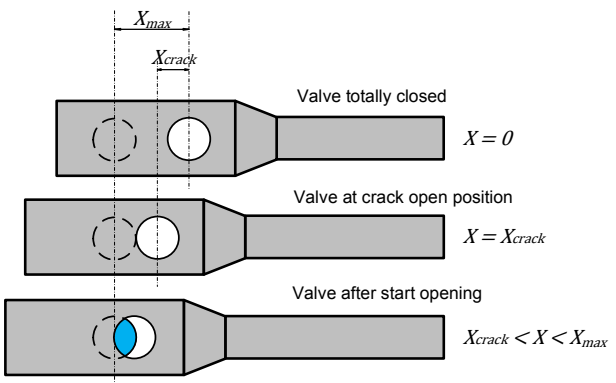

Figure 5: Representation of the opening area in a gate valve according to its position.

Finally, the assembled SVA and valve fixture shall be mounted in controllable hyperbaric environment, where the tests shall be carried out. A control module shall simultaneously control the force in the valve fixture and the function 
cycle in the SVA while records the data from the instruments.

\subsection{Test bench}

The hyperbaric chamber available at the Bremen University in Bremen, Germany, was employed to emulate the hyperbaric environment due to the column of water.

The valve fixture was realized with a mechanically coupled load hydraulic cylinder (MM201. The force profile is then applied by controlling its pressure differential with a proportional valve (QM101) powered by its own hydraulic power unit. This separated hydraulic circuit is partly embedded in the pressure chamber using pressure-sealed components. This prevents the pressure in the hyperbaric chamber disturbing the load control. A schematic of the test bench design is illustrated in Figure 6 .

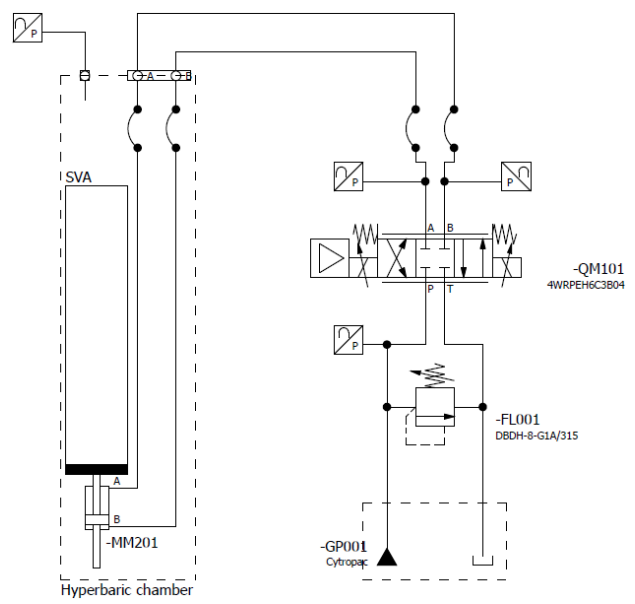

Figure 6: Schematic diagram of the test bench.

The test bench controller consists of an industrial PLC connected to both the instruments and the proportional valve of the load cylinder, and the electrical interface of the SVA running through a pressure-tight fitting located in the hyperbaric chamber lid.

The control architecture implemented to execute the test is shown in Figure 7. The SVA position controller is implemented as a finitestate machine. It receives the test configurations from the user and, according to its state, generates the reference for the SVA velocity controller, which, in turn, is implemented and tuned as a proportional-integral controller. The velocity controller drives the servomotor in the SVA to maintain the work cylinder velocity equal to the reference velocity. Simultaneously, the load force model calculates the differential pressure reference in the load cylinder according to the SVA position. This feeds yet another proportional-integral controller, whose action modulates the opening in the proportional valve, regulating the pressure in the load cylinder. Finally, to mitigate the disturbance of the SVA movement in the load force due to pressure imbalance in the load cylinder - a phenomenon that would not happen with a purely mechanical force in a real application - the SVA velocity is supplied to a feed-forward controller, which compensates the control effort in the proportional valve.

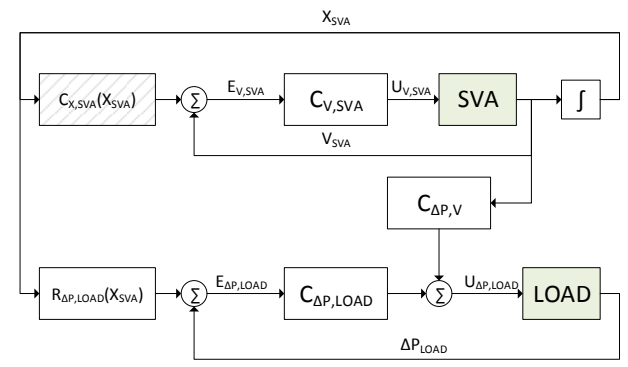

Figure 7: Test bench control architecture.

Because of the test bench layout, another three additional forces were included in the load force model. This is to compensate the friction force of the load cylinder $\left(F_{f, l o a d}\right)$, the force caused by the hydrostatic pressure acting on the load cylinder $\operatorname{rod}\left(F_{e, \text { load }}\right)$, and the weight force $\left(F_{W}\right)$ of the moveable parts, which are now vertically positioned with the valve fixture underneath the actuator. All friction forces, drag forces and the weight force depend on the movement direction. The pressure differential reference for the load cylinder $\left(R_{\Delta p, \text { load }}\right)$ is then given by the sum of the mentioned forces divided by the load cylinder effective area $\left(A_{\text {load }}\right)$, as shown in Equation 1.

$R_{\Delta p, \text { load }}=\frac{F_{v} \pm F_{\text {seal }} \pm F_{\text {drag }} \pm F_{f, \text { load }}-F_{e, \text { load }} \pm F_{W}}{A_{\text {load }}}$

In Figure 8, there is a depiction of the SVA coupled with the load cylinder during commissioning in the pressure chamber. 


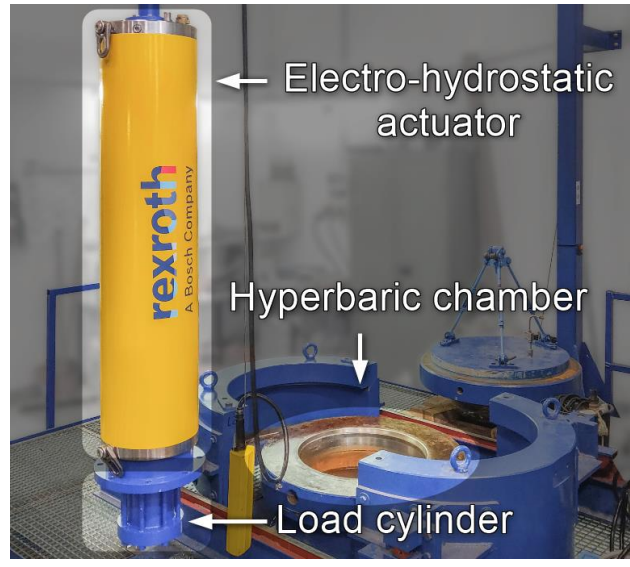

Figure 8: Test bench during commissioning.

A software was programmed to execute the regular actuation function and the fail-safe close function cycles.

The regular actuation function cycle consists of enabling the load controller, regulating the force on the fixture until within a tolerance of the reference. Then the SVA safety valves are closed and the velocity controller is enabled, driving the valve until the open position. There, the velocity controller stops the drive and waits 2 seconds to avoid dynamic distortions on the process variables, after which the velocity controller begins to drive the valve back to the closed position. The load controller continually regulates the load force to maintain the model reference force. When the actuator is back on the closed position, a completed cycle is accounted. After all cycles are completed, the safety valves are open and the spring chamber is released.

The fail-safe close function cycle is similar to the regular actuation up until the valve is open. Then, the velocity controller shuts down and the safety valves turned off, releasing the spring chamber. The load controller keeps regulating the force while the spring force passively brings the valve to the closed position. Once the closed position is reached, the cycle is counted as complete.

The hyperbaric chamber controls cannot be automated, thus the pressurizationdepressurization cycles have to be executed manually. The pressure in the chamber can be finely controlled via the user interface, the pressurization rate, however, cannot. In spite of that, the chamber is instrumented, so the cycles can be repeated on different conditions to achieve the qualification requirement.

\section{RESULT ANALYSIS}

The trials in hyperbaric chamber were executed over the course of six non-continuous days, in which the actuator and valve fixture were continuously immersed in the chamber filled with sink water for fourteen days. During the experiment sessions, the actuator was submitted to the equivalent pressure of the maximal rated depth for extended lengths of time, the longest lasting 8 hours and 36 minutes. This corresponds to $143.6 \%$ the time required by norm. During all trials, there was no loss of functionality observed. The internal pressure was steadily kept above the environment pressure, which asserts the structural integrity of the equipment.

\subsection{Regular actuation function}

\section{Cycles in hyperbaric environment}

To achieve the qualification conditions for the regular actuation function in hyperbaric environment, as previously declared in the test objectives, the hyperbaric chamber was kept at an average pressure of 332.3 bar $(\sigma=15.0$ bar $)$ throughout all cycles, or $110.7 \%$ the pressure at maximum rated depth. The average force (disregarding $F_{e, \text { load }}$ for comparison) throughout the cycles in the valve fixture before crack open - where the load is at its peak - was $101.2 \mathrm{kN}$ ( $\sigma$ $=0.1 \mathrm{kN}$ ). This deviation of the average of less than $2 \%$ from the reference value is deemed acceptable and demonstrates the controller overall effectiveness, asserting the validity of the test conditions as well. In these circumstances, 300 cycles were executed in total, $150 \%$ of the amount required for qualification.

In Figure 9 (time axis deliberately omitted), two full cycles are exhibited. It can be noticed a delay before the actuator starts moving after the test initialization. This corresponds to the spring chamber being latched. The compression can be seen indirectly as the SVA force rises linearly. Once latched, the actuator can move back and forth without having to push the spring chamber every cycle. Although the abrupt changes in the load force, the system position derivative does not see to change at all, which highlights a robust behavior towards load variations. The difference 
between the SVA force and the load force during the opening is caused by the friction between the hydraulic cylinder piston and rod and its seals. During the close motion, the valve force actually helps the movement. However, to prevent any uncontrollable outcome, the SVA dissipates the additional force in the piloted valves.

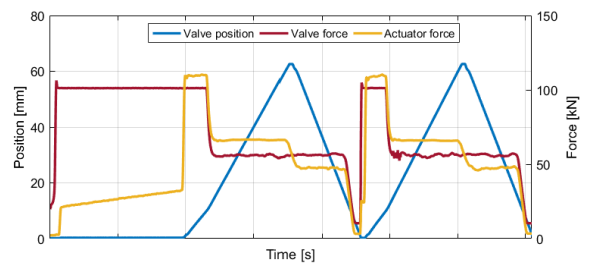

Figure 9: Actuator and load force and position in hyperbaric environment.

The system endurance is evaluated by averaging the load force as well as the SVA velocity and force were in their steady states before and after the crack-open during the opening motion for all cycles. These operational ranges were selected because correspond to the most demanding parts of the functional cycles. Figure $\mathbf{1 0}$ shows these values through the cycles.

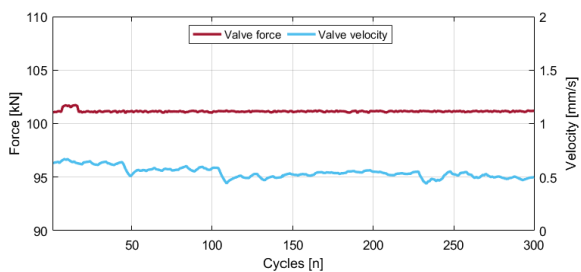

Figure 10: Average SVA velocity and load force during opening motion before crack open throughout all cycles in the hyperbaric environment.

\section{Cycles in atmospheric pressure environment}

The cycles in atmospheric pressure were executed after the tests in the hyperbaric chamber. In total, 610 cycles were executed, $152.2 \%$ of the required for qualification. Instead of executing these cycles without load as required, the same load condition equivalent to $10000 \mathrm{psi}$ through the valve fixture was applied in all of the cycles as well. Considering all cycles in atmospheric pressure, the average force applied by the valve fixture during opening before crack open was equal to $103.9 \mathrm{kN}(\sigma=$ $0.03 \mathrm{kN}$ ), again, less than $2 \%$ deviation from the reference value. In Figure 11, it is depicted two full cycles under the aforementioned conditions.

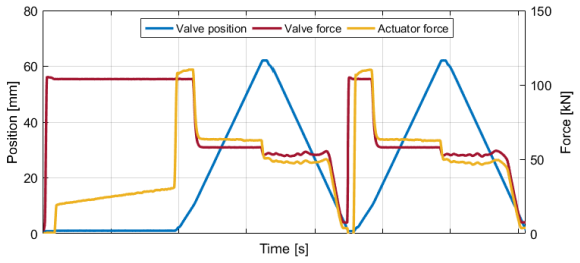

Figure 11: Actuator and load force and position in atmospheric pressure environment.

In comparison with the cycles executed in hyperbaric condition, the internal friction in the actuator observed in atmospheric pressure was $41.1 \%$ lower. This phenomenon is described by Suisse [12], where it is stated that, as the applied pressure increases, the friction forces between the dynamic seals and the cylinder piston and rod increases symmetrically, due to a higher normal force.

The SVA endurance was evaluated in atmospheric pressure in the same manner as it was in hyperbaric environment. Figure 12 depicts the averaged load force and SVA velocity during opening before crack open throughout all cycles.

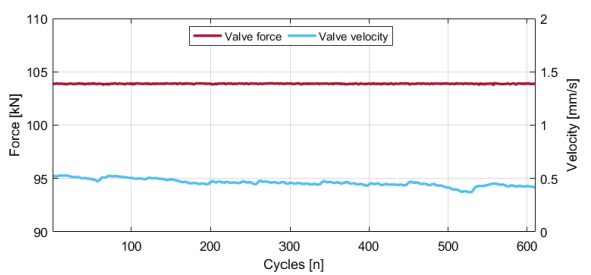

Figure 12: Average SVA velocity and load force during opening motion before crack open throughout all cycles in atmospheric pressure environment.

The steady decrease in the average velocity suggests a degradation of the volumetric efficiency, since the velocity is controlled but the input power is limited. This decrease was expected, most likely because the internal seals particularly in the hydraulic pump - degrade with time due to the compression set of the work pressure [13]. Nonetheless, this does not compromise the opening time, as the controller can be configured to compensate the setback after the crack open, where the load is significantly lower with sufficient power available to drive the valve faster. 


\subsection{Fail-safe close function}

\section{Cycles in hyperbaric environment}

In total, 30 cycles of the fail-safe close function were executed in hyperbaric environment. Half of the cycles were executed without load and the other half with the load equivalent of $10000 \mathrm{psi}$ pressure differential in the valve fixture. When executing the cycles without load, during the amount required by norm, the average pressure in the hyperbaric chamber was 333.0 bar $(\sigma=0.7$ bar). This corresponds to $111 \%$ the pressure at maximum rated depth. On the cycles with load, during the cycles required by norm, the average pressure was 341.8 bar ( $\sigma=1.1$ bar), or $113.9 \%$ the pressure at maximum rated depth. Counting the additional cycles executed for internal qualification, this amounts to $500 \%$ the number of required cycles for each condition.

The valve position and load force during the fail-safe close from one of the cycles with load is depicted in Figure 13. It can be seen that, during the closing motion, the resultant load force acts in the same direction, pushing the movement at a faster rate than the spring piston. At the crack open position, the friction force increases sharply, to which the resulting force from the load alone cannot finish the remaining stroke. The spring piston catches up after a few seconds, finishing the close movement. Considering all cycles, the average time for complete close with the load was 16.9 seconds ( $\sigma=0.1$ seconds), $43.60 \%$ less than the maximum tolerable time according to design requirements. The fastest cycle closed in 16.6 seconds, while the slowest finished in 17.1 seconds.

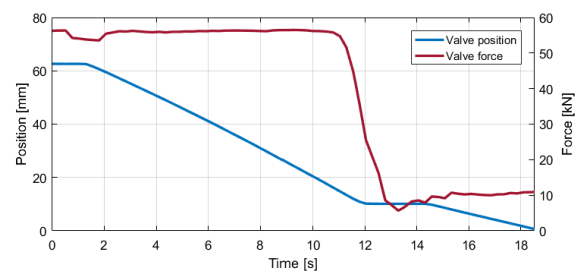

Figure 13: SVA and load actuator force and position through time in hyperbaric environment.

In the cycles without load, the closing movement is pushed entirely by the spring piston. This can be seen from the valve position during the closing of one of the cycles in Figure 14, which indirectly shows as the spring force decreases while relaxing. Considering all cycles, the average time for complete close without load was 24.5 seconds ( $\sigma=0.4$ seconds), $18.2 \%$ less than the maximum tolerable time. The fastest of these cycles closed in 23.9 seconds, the slowest in 25.6 seconds.

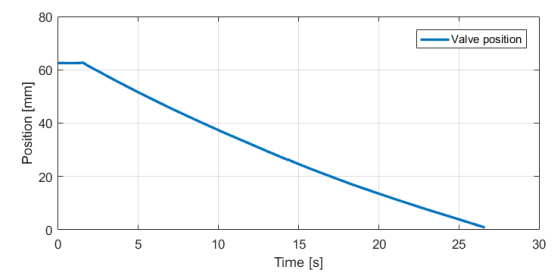

Figure 14: Valve position during fail-safe close without load in hyperbaric environment.

\section{Cycles in atmospheric pressure environment}

Another 30 cycles were correspondingly executed at an atmospheric pressure environment, again half of those with the prescribed load and half without it. The fail-safe close function did not present significant dynamic divergences from the tests ran in the hyperbaric chamber. However, due to the reduced internal friction, the average time to close was slightly smaller on both conditions.

The average time to close with load was 15.3 seconds $(\sigma=0.1$ seconds), $49.1 \%$ less than the maximum tolerable time and $9.5 \%$ faster than the average obtained under hyperbaric environment. The fastest cycle closed in 15.1 seconds and the slowest in 15.5 seconds. Without load, the average time to close was 20.5 seconds ( $\sigma=0.1$ seconds), $31.7 \%$ under the threshold and $16.3 \%$ lower than the same indicator obtained in the hyperbaric condition. The fastest cycle finished in 20.3 seconds, the slowest in 20.7 seconds.

\subsection{Pressure compensation function}

The actuator has been submitted to twice the amount of required cycles of pressurization and depressurization. Due to facility limitations, as aforementioned, the intensity of the pressurization and depressurization rates could be only marginally controlled. Therefore, the measurements have relatively higher standard deviations. Half of the cycles were executed with a relatively moderate rate and the other half with a more aggressive rate.

The most moderate pressurization cycle had already on average a pressurization rate of 55.6 
$\mathrm{bar} / \mathrm{min}(\sigma=12.4 \mathrm{bar} / \mathrm{min})-231.7 \%$ of the required by norm - and a depressurization rate of $71.0 \mathrm{bar} / \mathrm{min}(\sigma=33.6 \mathrm{bar} / \mathrm{min}), 197.2 \%$ of the required by norm. The average pressurization rate inside the EHA was $55.2 \mathrm{bar} / \mathrm{min}(\sigma=12.6$ $\mathrm{bar} / \mathrm{min}$ ) only $0.8 \%$ slower than the hyperbaric chamber, whereas its depressurization rate of $70.6 \mathrm{bar} / \mathrm{min}$ was $0.6 \%$ slower. This negligible delay is mostly caused by the dynamic response of the mechanical components of the pressure compensator. In Figure $\mathbf{1 5}$ it is depicted the internal pressure and the position of one of the pressure compensator units in respect to the external pressure during this particular cycle.

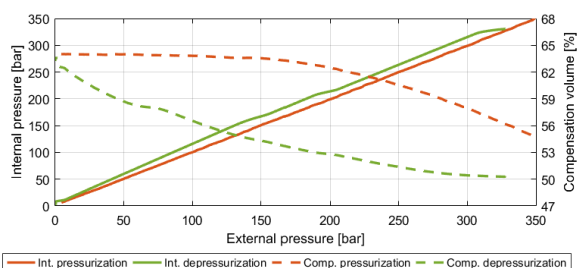

Figure 15: Compensator volume and internal pressure during a moderate pressurizationdepressurization cycle.

In the most aggressive pressurization cycle, the average pressurization rate was $103.7 \mathrm{bar} / \mathrm{min}(\sigma$ $=5.1 \mathrm{bar} / \mathrm{min}$ ), $432.1 \%$ of the required by norm. The average depressurization rate was 327.6 $\mathrm{bar} / \mathrm{min}$ with a brief maximum rate of 2647.2 $\mathrm{bar} / \mathrm{min}$ and minimum rate of $38.4 \mathrm{bar} / \mathrm{min}$ towards the end, the average being equivalent to $910 \%$ of the required by norm. In Figure 16 the internal pressure and the position of one of the pressure compensator units in respect to the external pressure during this cycle is plotted. In this case, the actuator presented on average a pressurization rate of $103.4 \mathrm{bar} / \mathrm{min}(\sigma=4.2$ $\mathrm{bar} / \mathrm{min}), 0.3 \%$ slower than the chamber and a rate of $309.5 \mathrm{bar} / \mathrm{min}(\sigma=99.7 \mathrm{bar} / \mathrm{min})$ during depressurization, $5.5 \%$ slower than the chamber. This shows as a hysteresis behavior, when the compensator begins to saturate its dynamic response to abrupt variations in the environmental pressure. Nonetheless, such extreme conditions are not realistically feasible in a real application and serves mostly to demonstrate the robustness of the system.

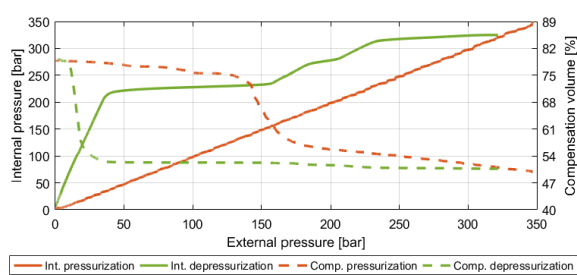

Figure 16: Compensator volume and internal pressure during an aggressive pressurizationdepressurization cycle.

\section{CONCLUSION AND OUTLOOK}

A novel concept of an electro-hydrostatic actuator (EHA) for subsea gate valves was presented, highlighting its several advantages over current technologies in this branch. The introduction of such systems in the market has the potential to drive considerably down the overall costs in oil and gas production, for instance. However, due to the stern reliability requirements of this industry, a series of experiments have to be executed in order to qualify this equipment before introducing it in the market. Part of these experiments require functionality validation under hyperbaric conditions.

In this paper, the qualification requirements, mostly relevant for hyperbaric conditions, were assessed and summarized as test objectives. Then a test procedure was devised and realized in a test bench, which was proven suitable to reproduce the required test conditions accurately. Yet, a remark to improve the reproducibility in future hyperbaric tests is to employ a hyperbaric chamber with an autonomous control of its pressurization-depressurization rates.

The prototype underwent a series of trials to achieve all qualification requirements. To meet the quality standards at Bosch Rexroth, these requirements were increased in all relevant aspects: actuator load, external pressure and amount of cycles. The results were then treated to guarantee proper statistical significance. In respect to all of its functions, the test subject exhibited a consistent robust performance even under the most unrealistic conditions. It has been noticed, as expected, a mild degradation on the volumetric efficiency throughout the endurance cycles and a variation of the friction forces in the hyperbaric environment. These factors, however, did not disturb the functional performance of the device. 
With these results, this actuator prototype already meets nearly all the criteria for technology readiness level 4 , according to API $17 \mathrm{~N}$ [14]. To complete its qualification process, additional functional validation tests are currently being executed in a temperature-controlled environment. Thereafter, the concept shall be considered sufficiently mature to be commissioned in the field.

\section{ACKNOWLEDGMENTS}

We would like to acknowledge and express our gratitude to the Laboratory of Hydraulic and Pneumatic Systems (LASHIP) at the Federal University of Santa Catarina, Brasil; to Bosch Rexroth AG, Germany; to the Center for Marine Environmental Sciences (MARUM) at Bremen University, Germany; to DNV-GL auditors and to all partners directly or indirectly involved. This study was made possible only through the collaboration of all these parties.

\section{LIST OF SYMBOLS}

$\begin{array}{ll}A_{\text {load }} & \text { Effective area of the load cylinder } \\ F_{c y l l} & \text { Hydraulic force in the work cylinder } \\ F_{c y l 2} & \text { Hydraulic force in the spring cylinder } \\ F_{d r a g} & \text { Friction force between gate and valve seats } \\ F_{e, S V A} & \text { Enclosure force } \\ F_{e, l o a d} & \text { Hydrostatic force caused by pressure acting } \\ & \text { on the load cylinder rod } \\ F_{f, l o a d} & \text { Friction force of the load cylinder } \\ F_{f, S V A} & \text { Friction forces in the SVA } \\ F_{W} & \text { Weight force of the SVA and load cylinder } \\ & \text { piston and rods } \\ \sigma & \text { Standard deviation } \\ F_{\text {seal }} & \text { Friction force between the bonnet seals and } \\ & \text { the stem } \\ F_{s p r i n g} & \text { Fail-safe spring force } \\ F_{v} & \text { Stem expulsion force due to the valve } \\ & \text { internal pressure } \\ C_{X, S V A} & \text { Position controller of the SVA } \\ C_{V, S V A} & \text { Velocity controller of the SVA } \\ C_{\Delta p, l o a d} & \text { Pressure controller of the load cylinder } \\ C_{\Delta p, V} & \text { Feed-forward controller to compensate SVA } \\ & \text { velocity disturbance in the load force } \\ R_{\Delta p, l o a d} & \text { Reference of pressure of the load cylinder } \\ X_{S V A} & \text { SVA position signal } \\ V_{S V A} & \text { SVA velocity signal } \\ \Delta p_{\text {load }} & \text { Load cylinder pressure differential signal } \\ U_{X, S V A} & \text { SVA motor control signal } \\ U_{\Delta p, l o a d} & \text { Load proportional valve control signal }\end{array}$

\section{REFERENCES}

[1] Berven, J (2013) Subsea production control systems for all-electric Xmas trees. Master thesis, Universitetet i Stavanger, Norway.

[2] Abicht, D.; Halvorsen, G.; Ramberg, R. M. (2017) Subsea all-electric. Paper presented at Offshore Technology Conference 2017, Houston, 1 - 4 May 2017.

[3] Weber, J. et al. (2016) Novel system architectures by individual drives. Paper presented at $10^{\text {th }}$ International Fluid Power Conference, Dresden, 8 - 10 March 2016.

[4] Alle, N. et. al. (2016) Review on Electro Hydrostatic Actuator for Flight Control. In: International Journal of Fluid Power, Issue 17(2): $125-145$.

[5] Orth, A.; Hendrix, G. (2018) An electromechanical actuator with hydrostatic drive for subsea trees to reduce CAPEX and OPEX with higher reliability and safety levels. Paper presented at Offshore Technology Conference 2018, Houston, 30 April - 3 May 2018.

[6] Mashiba, M. H. D. S. (2011) A influência dos parâmetros de operação e projeto no desempenho de atuação hidráulica de válvulas submarinas do tipo gaveta. Master thesis, Universidade Federal do Rio de Janeiro, Brasil.

[7] Kleppmann, W. $\left(8^{\text {th }}\right)(2013)$ Versuchsplanung: Produkte und Prozesse optimieren. Hanser, Munich.

[8] European Committee for Standardization (2009) EN ISO 10423 Petroleum and natural gas industries - Drilling and production equipment Wellhead and Christmas tree equipment. Belgium.

[9] American Petroleum Institute (2017) API Standard 17F Standard for subsea control systems. USA.

[10] Goularte, R. M.; Silva, J. P. D.; De Negri, V. J.; Orth, A. (2018) Static and dynamic performance analysis of an electro-hydrostatic system for ultra-deep water. Paper presented at Rio Oil \& Gas 2018, Rio de Janeiro, 24 - 27 September 2018.

[11] European Committee for Standardization (2011) EN ISO 13628-4 Petroleum and natural gas industries - Design and operation of subsea production systems - Part 4: Subsea wellhead and tree equipment. Belgium.

[12] Suisse, B. (2005) Research for dynamic seal friction modeling in linear motion hydraulic 
piston applications. Master thesis, The University of Texas at Arlington, Texas, USA.

[13] Plácido Neto, A. (2018) Failure distribution analysis of a novel subsea valve actuator concept based on reliability database. Paper presented at 10th IFAC Symposium on Fault Detection, Supervision and Safety for Technical Processes, Warsaw, 29 - 31 August 2018.

[14] American Petroleum Institute (2017) API Recommended practice $17 \mathrm{~N}$ Recommended practice on subsea production system reliability, risk and integrity management. USA. 